\title{
Chemical Enhancement of the Surface Enhanced Raman Scattering Signals of Anilines via Their Ortho-Substituents
}

\author{
Rachel A. Davies, Ngee S. Chong*, Beng Guat Ooi* \\ Department of Chemistry, Middle Tennessee State University, Murfreesboro, USA \\ Email: Ngee.Chong@mtsu.edu, *Beng.Ooi@mtsu.edu
}

Received May 29, 2013; revised June 31, 2013; accepted July 27, 2013

Copyright (C) 2013 Rachel A. Davies et al. This is an open access article distributed under the Creative Commons Attribution License, which permits unrestricted use, distribution, and reproduction in any medium, provided the original work is properly cited.

\begin{abstract}
While Raman spectroscopy is a useful method for analyzing many organic compounds, it is limited by relatively low sensitivity. Therefore, Surface Enhanced Raman Spectroscopy (SERS) based on the adsorption of organic analytes onto gold or silver nanostructures has been used to enhance the signal of chemicals presented at very low concentrations. Although the plasmonic effect of SERS has been shown to play a large role in signal enhancement, the significance of the chemical effect due to the analyte chemisorption on the gold or silver surface is less well understood. In this study, the role of aniline substituents is examined by probing the SERS intensities of various anilines in silver and gold colloids using a Raman spectrometer with an excitation wavelength of $785 \mathrm{~nm}$. The SERS enhancement factors and detection limits for aniline and its mono- and di-substituted ortho derivatives are determined and compared. Both the steric requirements of chemisorption and the inductive effects of electron-withdrawal due to the substituents affect the signal intensities of various vibrational modes of the amino group and the aromatic ring. The degree of enhancement is also related to the methods for preparing the silver and gold colloids, which are characterized by probing the nanoparticle morphology and its degree of aggregation using transmission electron microscopy (TEM).
\end{abstract}

Keywords: SERS; Raman; Enhancement Factors; Aniline Derivatives; Ortho-Substituent; Nanoparticle; TEM Characterization

\section{Introduction}

The SERS effect for amplifying the Raman signal via analyte adsorption onto gold or silver is based on two separate phenomena of electromagnetic and chemical enhancement. The electromagnetic enhancement effect based primarily on the excitation of localized surface plasmons in metallic nanostructures has been reported to be more significant than the chemical enhancement as a result of chemisorption via specific functional groups of the adsorbed molecules [1]. Many studies have focused on the investigation of the plasmonic effect based on the most SERS-active compounds, such as Rhodamine-6-G and Malachite Green [2,3], and have demonstrated SERS enhancement factors of greater than $10^{6}$. The chemical enhancement effect, based on the molecular structures and the functional groups of the adsorbed compound, is not as well understood, and is probed in this research study. Aniline, as an aromatic amine, is a useful compound for demonstrating the significance of the chemical

\footnotetext{
"Corresponding authors.
}

enhancement effect. This is due to interactions between the noble metal and the lone pair of electrons on the amino group, as well as interactions between the aromatic ring and the metal. Previous studies have identified both electron withdrawing character and steric hindrance as affecting chemical enhancement by the addition of substituent groups in the ortho, meta, and para positions [4]. The position of the substituent group on the ring will impact the electron withdrawing strength, the dipole moment of the molecule and also its induced dipole moment. Since both the inherent dipole and the induced dipole moment of a molecule are associated with their polarizability due to the electric field produced by the surface plasmon of the metal, the orientation of the adsorbed molecules and their vibrational modes are greatly affected in SERS. Calculation conducted for $\mathrm{o}_{-}, \mathrm{m-}$, and $p$-nitroaniline using the density functional theory (DFT) method have predicted that $o$-nitroaniline and $p$-nitroaniline have different adsorption orientations on the metal [5] due to the position of the nitro group on the aromatic ring. Both computational modeling and SERS studies of anilines have focused primarily on anilines 
with para-substituents [6-8] because of the industrial importance of anilines. In order to elucidate the role of chemical enhancement due to different functional groups in the ortho position of anilines, SERS studies are conducted for investigating how the steric and electronic effects of the ortho substituents would influence the charge transfer between specific anilines and metal through their interactions between the amino group or the aromatic ring of aniline and the metal. The magnitude of the SERS enhancement factors for various ortho-substituted anilines can be attributed to strength of the charge transfer interactions between the adsorbed analytes and the silver or gold colloids and correlated to affinity for electron donation based on their $\mathrm{pKa}$ values. The outcome of this study will contribute to the possible application of SERS for environmental monitoring of anilines.

\section{Materials and Methods}

\subsection{Reagents}

Aniline (99.5\% purity), 2-tert-butylaniline (99\% purity), 2-isopropylaniline (97\% purity), 2-chloroaniline (99.5\% purity), 2-nitroaniline (98\% purity), 2-fluoroaniline (99\% purity), 2-bromoaniline ( $98 \%$ purity), 2-iodoaniline ( $98 \%$ purity), 2,6-diisopropylaniline ( $97 \%$ purity), 2,6-dinitroaniline (97\% purity), 2,6-difluoroaniline (97\% purity), 2,6-dichloroaniline (98\% purity), and 2,6-dibromoaniline (97\% purity) were purchased from Sigma-Aldrich (St. Louis, MO, USA) and used without further purification. The silver nitrate $(100 \%)$ and sodium hydroxide pellets for silver colloid synthesis were purchased from Fisher Scientific (Waltham, MA, USA) and the hydroxylamine hydrochloride (98.9\%) from J.T. Baker. The gold (III) chloride trihydrate salt $(>99.9 \%)$ for preparing the aqueous $\mathrm{HAuCl}_{4}$ solution and N-2-hydroxyethylpiperazine-N'-2-ethanesulfonic acid or HEPES $(99.5 \%)$ for gold nanostar synthesis were purchased from Sigma-Aldrich (St. Louis, MO, USA). Acetonitrile solvent was purchased from Fisher Scientific (Waltham, MA) and the water used in this study was deionized using a Purelab Ultra deionizer purchased from ELGA, a subsidiary of Veolia Water (Houston, TX, USA). The deionized water has a resistivity of greater than 18 megaohm-cm.

\subsection{Synthesis of Colloidal Gold and Silver}

Colloidal silver nanospheres were prepared using the Leopold and Lendl method [9] by rapidly mixing $90 \mathrm{~mL}$ of $1.11 \times 10^{-3} \mathrm{M}$ silver nitrate with $10 \mathrm{~mL}$ solution of 1.5 $\times 10^{-2} \mathrm{M}$ hydroxylamine hydrochloride and $3.0 \times 10^{-2} \mathrm{M}$ sodium hydroxide. The colloidal solution was stirred continuously for 15 minutes at room temperature and concentrated 5-fold using a Beckman Coulter Allegra 64R Centrifuge at $5000 \mathrm{rpm}$ for 15 minutes at $4^{\circ} \mathrm{C}$.
Colloidal gold nanostars were prepared by the Xie et al. method [10] with minor modifications. Nanostars were formed by the reduction of $115 \mu \mathrm{L}$ of $24.25 \mathrm{mM}$ gold (III) chloride solution by $100 \mathrm{mM}$ HEPES solution ( $\mathrm{pH} 7.5 \pm$ 0.5 , adjusted using $1 \mathrm{M} \mathrm{NaOH}$ ) at 2:3 volume ratio. The reaction was allowed to proceed without shaking or stirring for 20 minutes, during which a color change to colorless followed by dark purple occurred.

\subsection{Sample Preparation}

Based on the literature values of solubilities of aniline compounds in water, stock solutions of the following concentrations were prepared for aniline $(20,000 \mathrm{ppm}$ or $\mathrm{mg} / \mathrm{kg}$ ), 2-tert-butylaniline (400 ppm), 2-isopropylaniline (1800 ppm), 2,6-diisopropylaniline (150 ppm), 2-fluoroaniline (950 ppm), 2-chloroaniline (1200 ppm), 2-bromoaniline (700 ppm), 2-iodoaniline (425 ppm), 2,6-difluoroaniline (300 ppm), 2,6-dichloroaniline (100 ppm), 2,6dibromoaniline (75 ppm), 2-nitroaniline (650 ppm), and 2,6-dinitroaniline (50 ppm). Stock solutions for each compound were also prepared in acetonitrile at concentrations of $20,000 \mathrm{ppm}$ or $\mathrm{mg} / \mathrm{kg}$. The lower concentrations used for SERS measurements were prepared by diluting the stock solutions. Samples were prepared using a 1 to 9 volume ratio of analyte in acetonitrile to silver colloid and 1 to 1 volume ratio of analyte in deionized water to the colloidal gold nanostars. The aniline samples were also analyzed on Klarite ${ }^{\mathrm{TM} B}$ chips (Renishaw Diagnostics Ltd., Glasgow, UK) by carefully placing $1 \mu \mathrm{L}$ at a time using a $10 \mu \mathrm{L}$-syringe. The Raman spectra were collected while the sample was wet and also after it was dried. The laser excitation beam was focused at the periphery of the dried sample spots with a probeto-sample distance of $7 \mathrm{~mm}$.

\subsection{Conditions for Raman Spectroscopy}

Spectral measurements were performed using the ProRaman-L of Enwave Optronics, Inc. (Irvine, CA) at a wavelength of $785 \mathrm{~nm}$. The integration period for collecting the signal was varied from $1-120$ seconds, with the spectrometer setting of " 1 " for boxcar. The laser power was kept constant for comparative SERS and Raman measurements. The data were acquired using the EZRaman Reader V7.8.2 and analyzed using Thermo Electron's OMNIC 7.0 software. Figures of overlaid spectra were prepared using the "common scale" and "spectra offset" modes. The peak areas of the $1-3$ most intense SERS peaks were used for the calculation of SERS enhancement factors using the integration tool in OMNIC and peak-to-peak noise was used for calculation of the minimum detection limits of analytes based on the 3-sigma definition. 


\subsection{Transmission Electron Microscopy}

The TEM specimens for the characterization of particle size and morphology of colloidal gold nanostars were prepared as follows. Colloidal nanoparticles were nebulized using a Micro Mist Nebulizer, obtained from Hudson RCI-Teleflex Incorporated (Research Triangle Park, NC), for 40 - 60 seconds onto carbon-coated grids placed on either side of the rim of the cap with the nozzle. Alternatively, the carbon-coated grids were held with a pair of tweezers along the path traveled by the mist produced by the nebulizer at approximately $10 \mathrm{~cm}$ from the nozzle. These nebulization procedures allowed uniform deposition of gold and silver nanoparticles onto the carbon-coated grids. Transmission electron microscopy was performed using a Hitachi Model H-7650 TEM at an electron acceleration voltage of $80-100 \mathrm{kV}$.

\subsection{Data Analysis}

The SERS enhancement factors were calculated using the Equation (1) by comparing the relative sensitivity of each compound for SERS versus normal Raman signals for spectral signals associated with specific vibrational modes. The Raman spectrometer conditions of laser power and signal integration period were kept constant for both SERS and normal Raman measurements. For almost all spectral peaks of all compounds studied, the wavenumber of the peaks for a specific vibrational mode will be similar for both SERS and normal Raman spectra. However, in the case of the SERS signal for aniline, the vibration mode of $\mathrm{NH}_{2}$ wagging was shifted from 538 $\mathrm{cm}^{-1}$ in normal Raman to $989 \mathrm{~cm}^{-1}$ in SERS spectra based on computational modeling [6,11]. Therefore, only in the case of the $\mathrm{NH}_{2}$ wagging mode for aniline, the large peak shift (i.e. Raman to SERS) of $534 \mathrm{~cm}^{-1}$ to 989 $\mathrm{cm}^{-1}$ is accounted for in Equation 1 instead of the typical peak shift of less than $50 \mathrm{~cm}^{-1}$.

Enhancement Factor:

$$
\frac{\text { SERS Sample peak area }}{\text { Raman Std. peak area }} \times \frac{\text { Std. conc. } \mathrm{mg} / \mathrm{kg}}{\text { Sample conc. } \mathrm{mg} / \mathrm{kg}}
$$

The method for calculating the limit of detection (LOD) is shown in Equation (2). The LOD values shown in Table 1 are equivalent to the lowest analyte concentrations that gives peak areas corresponding to three times their respective background noise or standard deviation of the background signals.

Limit of Detection:

$$
\frac{3 \times \text { Background noise }}{\text { SERS Sample peak area }} \times \text { Sample conc. } \mathrm{mg} / \mathrm{kg}
$$

The pKa values in Table 2 were based on values reported in SciFinder Scholar from Chemical Abstract Services. All values reflected standard $\mathrm{pKa}$ for the proto- nated amino group at $25^{\circ} \mathrm{C}$.

\section{Results and Discussions}

\subsection{Comparison of Nanostructures for SERS}

The Raman and SERS spectra of aniline in colloidal silver nanospheres, gold nanostars, and Klarite ${ }^{\mathrm{TM}}$ gold substrates are compared in Figure 1. The three most prominent spectral peaks, labeled for each spectrum, were used to determine the enhancement factors tabulated in Table 2. The major peak of $989 \mathrm{~cm}^{-1}$ on the SERS spectra for aniline adsorbed onto silver can be attributed to the vibrational mode of $\mathrm{NH}_{2}$ stretch and corresponds to the 538 $\mathrm{cm}^{-1}$ peak in the normal Raman spectra for aniline. The spectral shift of $451 \mathrm{~cm}^{-1}$ for the $\mathrm{NH}_{2}$ wagging mode was verified by computational modeling using the DFT method of the Gaussian software [11]. The enhancement factor for the $\mathrm{NH}_{2}$ stretching vibrational mode is calculated to be $2.52 \times 10^{3}$. Other major SERS peaks were found at $995 \mathrm{~cm}^{-1}, 1175 \mathrm{~cm}^{-1}, 1234 \mathrm{~cm}^{-1}, 1593 \mathrm{~cm}^{-1}$, and $1613 \mathrm{~cm}^{-1}$ with a wavenumber uncertainty of $\pm 15 \mathrm{~cm}^{-1}$. The gold colloid was found to yield stronger signals than the silver colloid at the same aniline concentration. In general the enhancement factors for gold are better than those for silver as reported previously [12]. One possible explanation, based on work done by Zhao et al. [11], is the formation of a stronger charge transfer bond between aniline and the metal due to the optimal orbital overlap as a result of a smaller energy difference between electron donor (highest occupied molecular orbital or HOMO of aniline) and electron acceptor (lowest unoccupied molecular orbital or LUMO of metal) for gold compared to silver. Based on computational modeling of aniline adsorption on a metal cluster of four atoms, the energy gaps between HOMO and LUMO were found to be $1.55 \mathrm{eV}$ for aniline- $\mathrm{Au}_{4}$ and $2.69 \mathrm{eV}$ for aniline- $\mathrm{Ag}_{4}[11,13]$. Furthermore, the SERS signals based on gold colloid were

Table 1. Limits of Detection (LOD) for aniline and selected anilines in gold and silver colloids.

\begin{tabular}{ccc}
\hline Analyte & \multicolumn{2}{c}{ Limit of Detection (ppm) } \\
\cline { 2 - 3 } Aniline & Silver Colloid & Gold Colloid \\
\hline 2-tert-butylaniline & 1.6 & 0.82 \\
2-isopropylaniline & 25 & 9.7 \\
2,6-diisopropylaniline & 140 & 2.3 \\
2,6-difluoroaniline & 67 & 1.1 \\
2,6-dichloroaniline & 590 & 22 \\
2,6-dibromoaniline & 140 & 33 \\
2,6-dinitroaniline & 510 & 16 \\
\hline
\end{tabular}


Table 2. Comparison of SERS Enhancement Factors for Gold Nanostar and Klarite ${ }^{\mathrm{TM}}$.

\begin{tabular}{|c|c|c|c|c|c|}
\hline \multirow{3}{*}{ Analyte } & \multicolumn{3}{|c|}{ Wavenumber $\left(\mathrm{cm}^{-1}\right)$} & \multicolumn{2}{|c|}{ Enhancement Factor } \\
\hline & \multirow[t]{2}{*}{ Normal } & \multicolumn{2}{|c|}{ SERS Spectra } & \multirow[b]{2}{*}{ Colloid } & \multirow[b]{2}{*}{ Klarite $^{\mathrm{TM}}$} \\
\hline & & Colloid & Klarite $^{\mathrm{TM}}$ & & \\
\hline \multirow{5}{*}{ Aniline pKa 4.61} & 1611 & - & 1593 & - & $7.08 \mathrm{E}+03$ \\
\hline & 1186 & 1235 & - & $2.56 \mathrm{E}+04$ & - \\
\hline & 1165 & - & 1175 & - & $3.84 \mathrm{E}+04$ \\
\hline & 1005 & 996 & - & $3.73 \mathrm{E}+03$ & - \\
\hline & 534 & $531(996)^{\mathrm{a}}$ & $533(1175)^{\mathrm{a}}$ & $2.02 \mathrm{E}+04(4.56 \mathrm{E}+04)^{b}$ & $2.20 \mathrm{E}+04(7.64 \mathrm{E}+04)^{b}$ \\
\hline \multirow{3}{*}{ 2-tert-butylaniline pKa 3.78} & 863 & 865 & - & $6.57 \mathrm{E}+02$ & - \\
\hline & 686 & 682 & - & $3.29 \mathrm{E}+02$ & - \\
\hline & 546 & 547 & - & $2.52 \mathrm{E}+03$ & - \\
\hline \multirow{3}{*}{ 2-isopropylaniline pKa 4.42} & 1265 & 1239 & - & $4.31 \mathrm{E}+03$ & - \\
\hline & 1042 & 1022 & - & $1.91 \mathrm{E}+03$ & - \\
\hline & 599 & 593 & - & $2.62 \mathrm{E}+03$ & - \\
\hline \multirow{3}{*}{ 2,6-diisopropylaniline pKa 4.25} & 1451 & 1430 & 1447 & $6.49 \mathrm{E}+04$ & $3.56 \mathrm{E}+03$ \\
\hline & 1268 & 1251 & 1249 & $5.76 \mathrm{E}+03$ & $5.72 \mathrm{E}+01$ \\
\hline & 1122 & 1113 & 1136 & $7.24 \mathrm{E}+03$ & $1.53 \mathrm{E}+01$ \\
\hline \multirow{3}{*}{ 2-fluoroaniline pKa 3.20} & 1636 & 1614 & - & $1.31 \mathrm{E}+02$ & - \\
\hline & 1210 & 1235 & - & $7.16 \mathrm{E}+02$ & - \\
\hline & 1030 & 1040 & - & $9.20 \mathrm{E}+01$ & - \\
\hline \multirow{3}{*}{ 2-chloroaniline pKa 2.65} & 1602 & 1593 & - & $1.62 \mathrm{E}+01$ & - \\
\hline & 1036 & 1042 & - & $1.32 \mathrm{E}+01$ & - \\
\hline & 572 & 567 & - & $3.89 \mathrm{E}+01$ & - \\
\hline \multirow{3}{*}{ 2-bromoaniline pKa 2.54} & 1594 & 1590 & - & $4.25 \mathrm{E}+03$ & - \\
\hline & 1408 & 1389 & - & $1.11 \mathrm{E}+04$ & - \\
\hline & 1164 & 1178 & - & $6.93 \mathrm{E}+02$ & - \\
\hline \multirow{3}{*}{ 2-iodoaniline pKa 2.54} & 1588 & 1590 & - & $2.13 \mathrm{E}+02$ & - \\
\hline & 1013 & 1015 & - & $3.82 \mathrm{E}+02$ & - \\
\hline & 553 & 555 & - & $3.38 \mathrm{E}+02$ & - \\
\hline 2,6-difluoroaniline pKa 1.81 & 1296 & 1284 & - & $1.94 \mathrm{E}+01$ & - \\
\hline \multirow[t]{2}{*}{ 2,6-dichloroaniline $\mathrm{pKa} 0.71$} & 1099 & 1090 & - & $5.31 \mathrm{E}+02$ & - \\
\hline & 1460 & 1448 & 1444 & $3.49 \mathrm{E}+03$ & $2.33 \mathrm{E}+03$ \\
\hline \multirow[t]{2}{*}{ 2,6-dibromoaniline $\mathrm{pKa} 0.48$} & 1240 & 1250 & 1259 & $1.01 \mathrm{E}+03$ & $7.47 \mathrm{E}+02$ \\
\hline & 588 & 581 & 584 & $2.11 \mathrm{E}+02$ & $6.66 \mathrm{E}+00$ \\
\hline \multirow{3}{*}{ 2-nitroaniline $\mathrm{pKa}-0.23$} & 1290 & 1300 & - & $7.85 \mathrm{E}+00$ & - \\
\hline & 1249 & 1269 & - & $2.64 \mathrm{E}+00$ & - \\
\hline & 818 & 827 & - & $3.84 \mathrm{E}+01$ & - \\
\hline \multirow{3}{*}{ 2,6-dinitroaniline $\mathrm{pKa}-5.45$} & 1342 & 1344 & - & $6.88 \mathrm{E}-01$ & - \\
\hline & 1293 & 1295 & - & $1.95 \mathrm{E}-01$ & - \\
\hline & 821 & 823 & - & $6.41 \mathrm{E}-01$ & - \\
\hline
\end{tabular}

${ }^{\mathrm{a}}$ Numbers in brackets are for the associated SERS peak shifts due to the $\mathrm{NH}_{2}$ wagging vibrational mode based on Reference [11]; ${ }^{\mathrm{b}} \mathrm{Corresponding}$ enhancement factors based on the shift from $534 \mathrm{~cm}^{-1}$ to $996 \mathrm{~cm}^{-1}$ or $1175 \mathrm{~cm}^{-1}$. 


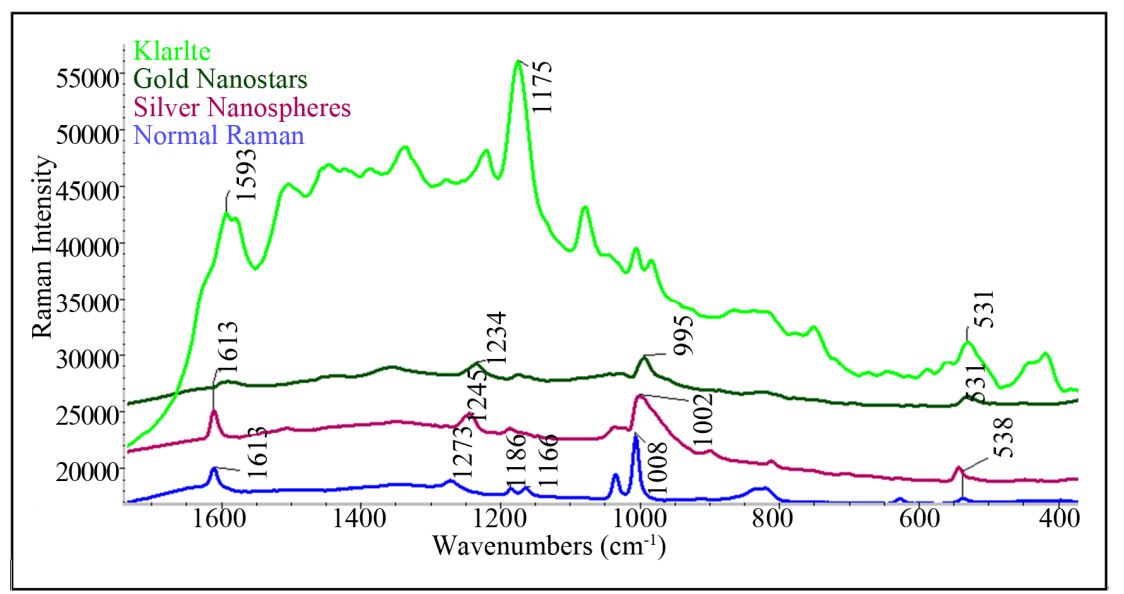

Figure 1. Normal and SERS Spectra of Aniline in Aqueous Solutions. The normal Raman spectrum was obtained for aniline at 20,000 ppm, whereas the SERS spectrum for aniline was obtained for a sample prepared by mixing $10,000 \mathrm{ppm}$ aniline with silver colloid at a 1: 9 volume ratio. The Klarite ${ }^{\mathrm{TM}}{ }^{\circledR}$ SERS spectrum was collected at the edge of a dried spot of $6 \mu \mathrm{L}$ of 6 ppm aniline solution. The SERS spectrum based on gold nanostars was obtained for a sample of 6 ppm aniline mixed with the colloid at a 1:1 volume ratio. All spectra were acquired for 120 seconds.

more consistent and reproducible whereas silver colloid tends to yield larger signal variation when different batches of colloid were used for SERS analysis. Besides, the silver colloid requires an extra step of pre-concentration to give larger signal enhancement.

The broad unresolved SERS peak of 989/996 $\mathrm{cm}^{-1}$ (wagging) for aniline in silver/gold colloids is the most prominent feature and has a "shoulder" in the vicinity of $1030 \mathrm{~cm}^{-1}$ due to the ring breathing mode. The peaks at $1602 \mathrm{~cm}^{-1}$ and $1234 \mathrm{~cm}^{-1}$ can be attributed to the $\mathrm{NH}_{2}$ scissoring and $\mathrm{CN}$ stretching, respectively. The SERS spectra for aniline in gold and silver colloids are very similar. However, for the SERS spectrum of aniline obtained with Klarite ${ }^{\mathrm{TM}}$, the ring deformation mode at 1175 $\mathrm{cm}^{-1}$ seems to dominate whereas the corresponding signals for the colloidal gold and silver samples are significantly weaker. Similar observation of spectral anomaly at $1175 \mathrm{~cm}^{-1}$ was also noted in an earlier study of 4-aminobiphenyl involving Klarite ${ }^{\mathrm{TM}}$ substrate [14]. The presence of the dominant peak at $1175 \mathrm{~cm}^{-1}$ is likely due to the strong interaction of the aromatic ring in aniline with the gold surface of the nanocrevices in the array of inverted pyramids on the Klarite ${ }^{\mathrm{TM}}$ substrate [15].

\subsection{The Role of Steric Effects on SERS Signals}

For aromatic amines such as aniline or 4-aminobiphenyl with unhindered interaction of the amino groups with gold or silver surfaces, the SERS enhancement factors due to the $\mathrm{NH}_{2}$ wagging and stretching modes are usually large. In this study, anilines with alkyl group(s) in the ortho position are investigated with regard to their roles in the SERS enhancement effects. As shown in Figure 2, it was found that both the intensities of the vibrational modes of $\mathrm{NH}_{2}$ wagging and scissoring at approximately
$996 \mathrm{~cm}^{-1}$ and $1602 \mathrm{~cm}^{-1}$ were diminished while the ring breathing mode at $1022 \mathrm{~cm}^{-1}$ became more pronounced. The $t$-butyl group was more effective in suppressing both the $\mathrm{NH}_{2}$ wagging and scissoring modes. Furthermore, the presence of two isopropyl groups at the ortho positions of aniline also prevents the molecule from contorting itself in forming a charge-transfer bond as is possible with 2-isopropylaniline.

\subsection{The Steric and Electron-Withdrawal Effects of Halogen Substituent Groups on SERS Signals of Anilines}

The electron withdrawing effects of halogens due to their different electronegativity values along with their trends in atomic radii is well known in chemistry. For the mono-halogenated anilines, the influence of electron withdrawal generally makes it less likely for the amino groups to interact or donate electrons to the silver or gold nanoparticles. So, as shown in Figure 3, the peak at the $989 \pm 5 \mathrm{~cm}^{-1}$ due to $\mathrm{NH}_{2}$ wagging mode is attenuated in all the mono-halogenated anilines and the $\mathrm{NH}_{2}$ scissoring mode at $1600 \pm 15 \mathrm{~cm}^{-1}$ shifts toward the smaller wavenumbers (i.e. $1613 \rightarrow 1593 \rightarrow 1590$ ) for 2-fluoroaniline to 2-bromoaniline. There is also a similar shift toward the lower wavenumbers for the $\mathrm{NH}_{2}$ wagging mode at $560 \pm 10 \mathrm{~cm}^{-1}$ region going from fluoro- to iodosubstituents. Other SERS spectral differences are due to the presence of carbon-halogen vibrational modes in the $1000-600 \mathrm{~cm}^{-1}$ region. The presence of both bromine and iodine substituents drastically decreases the analyticcal sensitivity of these aniline derivatives, resulting in their higher detection limits (Table 1).

The presence of two halogen substituents in both ortho positions of anilines further reduces the intensities of 


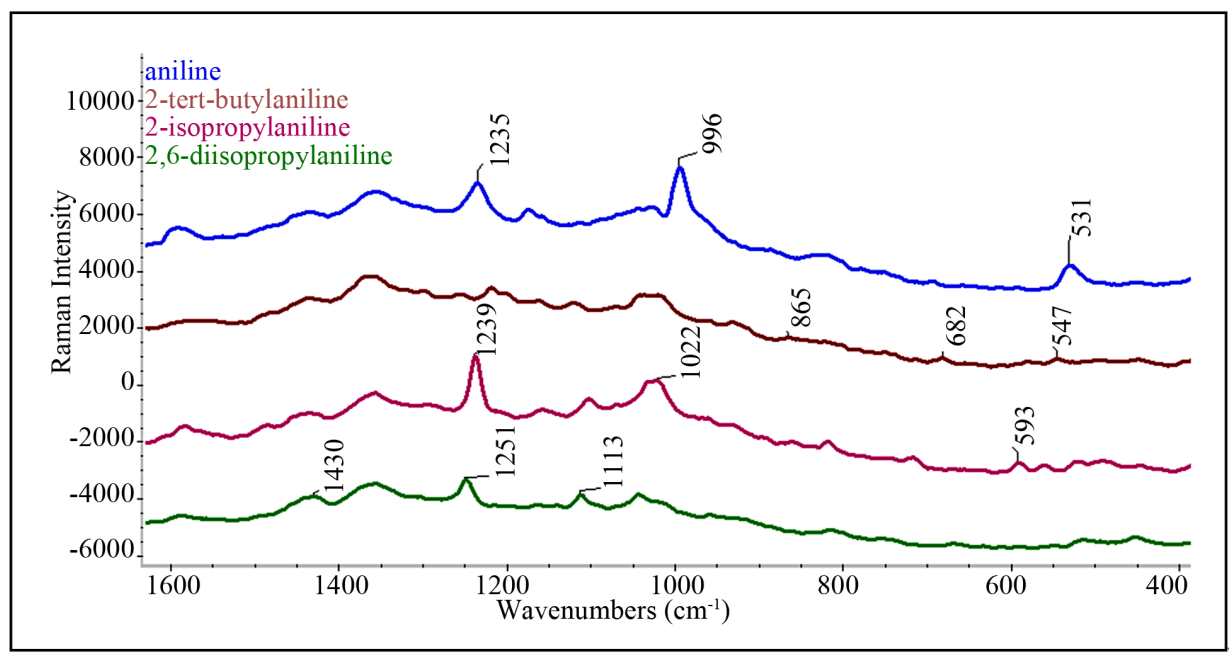

Figure 2. SERS Spectra of Aniline and Aniline Derivatives with Alkyl Substituents. Colloidal gold nanostars were mixed with aqueous standards at 1:1 volume ratio for Raman analysis with an integration time of 120 seconds. The initial concentrations of the standards were $6.0 \mathrm{ppm}$ for aniline, $10 \mathrm{ppm}$ for 2 -t-butylaniline, $12 \mathrm{ppm}$ for 2-isopropylaniline, and $3.0 \mathrm{ppm}$ for 2,6-diisopropylaniline.

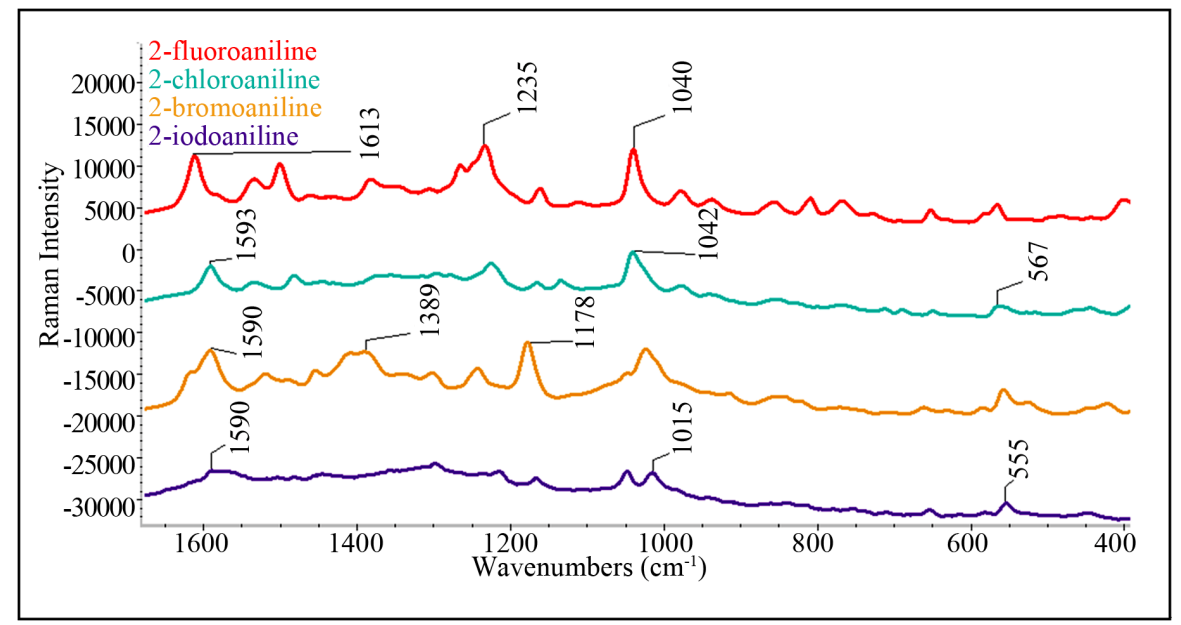

Figure 3. SERS Spectra of Mono-Halogenated Anilines. Spectra were obtained by mixing aqueous standards with gold colloid in 1:1 volume ratio for measurement using spectral integration time of 90 seconds. The initial concentrations of the standards were $950 \mathrm{ppm}$ for 2-fluoroaniline, $1200 \mathrm{ppm}$ for 2-chloroaniline, $700 \mathrm{ppm}$ for 2-bromoaniline, and $425 \mathrm{ppm}$ for 2-iodoaniline.

SERS signals while showing similar shift to lower wavenumbers for specific vibrational modes as shown in Figure 4. Besides showing a smaller Raman intensity scale relative to the mono-halogenated anilines, the dihalogenated aniline derivatives also shows higher fluorescence background.

\subsection{Influence of pKa Values on SERS Signals}

There is a strong correlation between $\mathrm{pKa}$ values of the analytes and their SERS enhancement factors measured in gold colloid (Figure 5). This effect was most clearly observed for the plot with data for aniline, three alkyl anilines, and its nitro and dinitro derivatives. Aniline $(\mathrm{pKa}=4.61)$ and diisopropylaniline $(\mathrm{pKa}=4.25)$, which have the two highest $\mathrm{pKa}$ values produced the highest enhancement factors whereas both 2,6-dinitroaniline ( $\mathrm{pKa}$ $=-0.23)$ and 2-nitroaniline $(\mathrm{pKa}=-5.45)$ with the lowest and second-lowest $\mathrm{pKa}$ values, have the lowest and second-lowest enhancement factors (EF), respectively. The ortho-substituents generally decrease the basicity of the amino group in aniline derivatives and therefore make it less likely for amino group to share its lone pair of electrons with $\mathrm{Ag} / \mathrm{Au}$ surface via the charge transfer bond. The trend line shows the equation of $\mathrm{EF}=$ $69.6 \mathrm{e}^{1.018(\mathrm{pKa})}$ with an $\mathrm{R}^{2}$ value of 0.927 for the linear regression analysis of the data plotted in logarithmic scale because of the large span of data values.

The nature of the aniline chemisorption on metals is 


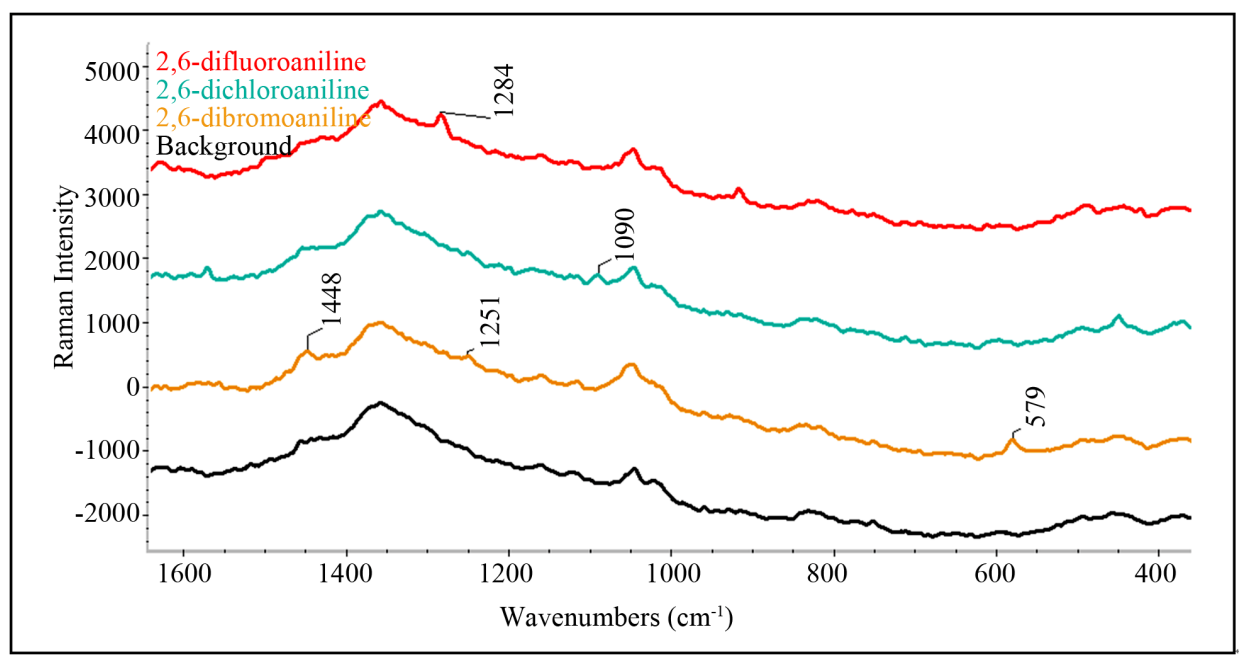

Figure 4. SERS Spectra of Di-Halogenated Anilines. Spectra were obtained for samples prepared by mixing aqueous standards with gold colloid in 1:1 volume ratio for spectral acquisition time of 90 seconds. The initial analyte concentrations were 300 ppm for 2,6-difluoroaniline, 100 ppm for 2,6-dichloroaniline, and 75 ppm for 2,6-dibromoaniline.

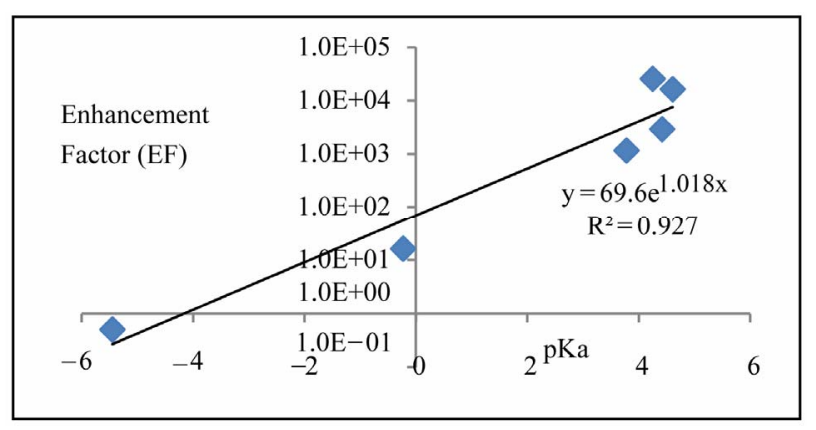

Figure 5. Plot of SERS Enhancement Factor versus pKa.

akin to the acid-base interaction between the electronrich lone pair on the basic amino group and the empty orbital behaving as a Lewis acid in accepting the lone pair on the metals. Therefore, it is reasonable to expect that $\mathrm{pKa}$ values of aniline and its derivatives to be related to their enhancement factors. However, it is also important to recognize that the degree of enhancement is also related to the compatibility of energy levels between the aniline derivatives and the metal surfaces. As alluded earlier, the SERS enhancement factors depend on the energy gap between the HOMO/LUMO levels of the analyte and metal surfaces. In addition, the steric factors of different substituent groups cannot be easily computed or modeled and taken into account along with the pKa values and the HOMO/LUMO values to give a complete understanding of how each variable affects the enhancement factor. It is beneficial to further explore computational modeling to predict the effects of various aniline substituents on their physical properties including acidity or pKa. Previous studies have shown that charge distribution in anilines and the charge density on the nitrogen of the amino group in anilines can affect the acidity of aniline and its derivatives $[16,17]$.

\subsection{TEM Characterization of Gold Nanostars}

The nanoparticle morphology in the gold or silver colloids greatly influences the magnitude of signal enhancement as previously noted for nanospheres, nanorods, nanowires, and nanostars [18]. The Xie's method [10] of reducing the gold (III) chloride solutionto gold nanoparticles by HEPES solution yielded a mixture of nanostars and nanospheres that are highly aggregated as shown in Figure 6(a). X-ray microanalysis was used to confirm the gold elemental signal of the nanoparticles. The numbers of nanoparticles for each aggregated cluster is significantly larger than those observed for silver colloids obtained by the method of Lendl and Leopold [9]. The nanoparticle size is in the range of $10-30 \mathrm{~nm}$ and the number of particles per cluster is estimated in the range of $20-60$, which is much larger than the nanosphere clusters of gold and silver reported earlier [14]. For the TEM image shown in Figure 6(a), about 5 7 distinct clusters were formed from the evaporation of the nebulized colloidal droplet diameter of $1-5 \mu \mathrm{m}$. This implies that all the particles observed in Figure 6(a) were contained in a one single aerosol droplet. As the aqueous solvent evaporated, the distinct clusters overlapped to varying extent. In the colloidal state, the cluster size of 20 - 80 nanoparticles provided "hot spots" for the localized plasmon effects to yield large SERS enhancement factors. The estimation of the ratio of nanostars to nanospheres on the 2-dimensional TEM image is difficult due to significant aggregation and stacking of nanoparticles.

In Figure 6(b), isolated nanostars along with nanospheres were imaged at 500,000-fold magnification and 


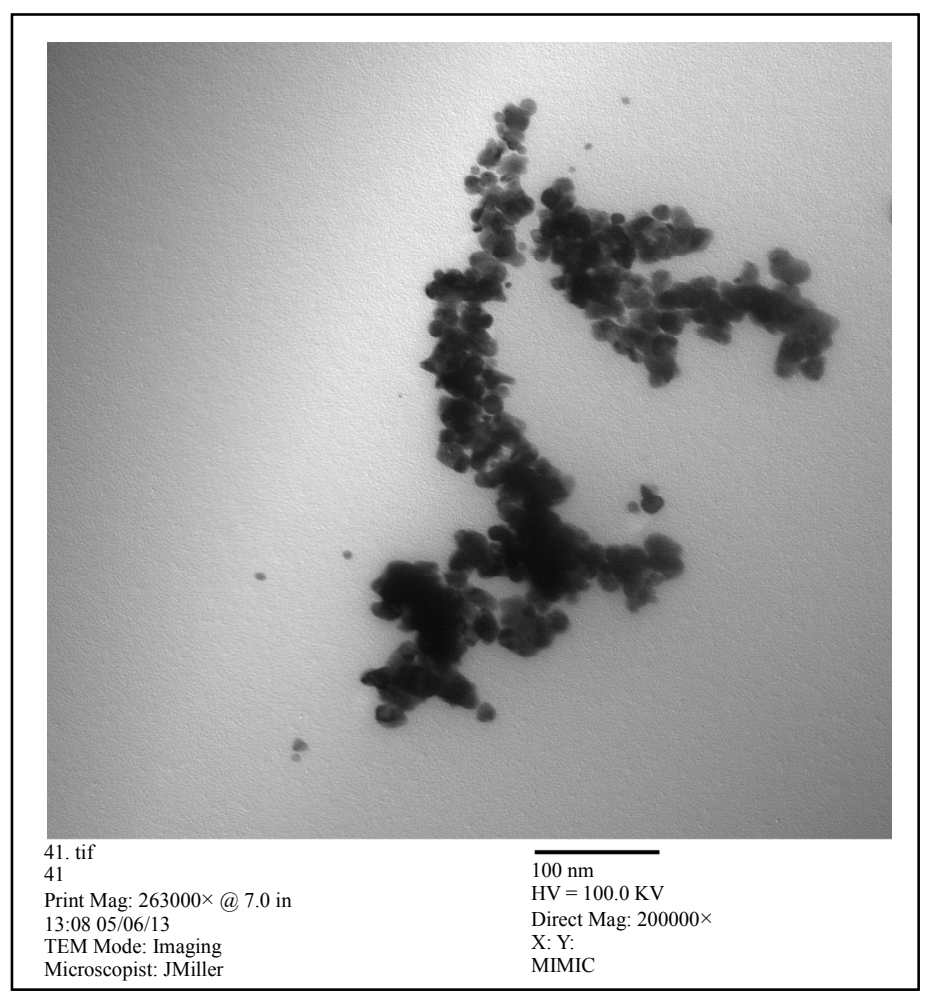

(a)

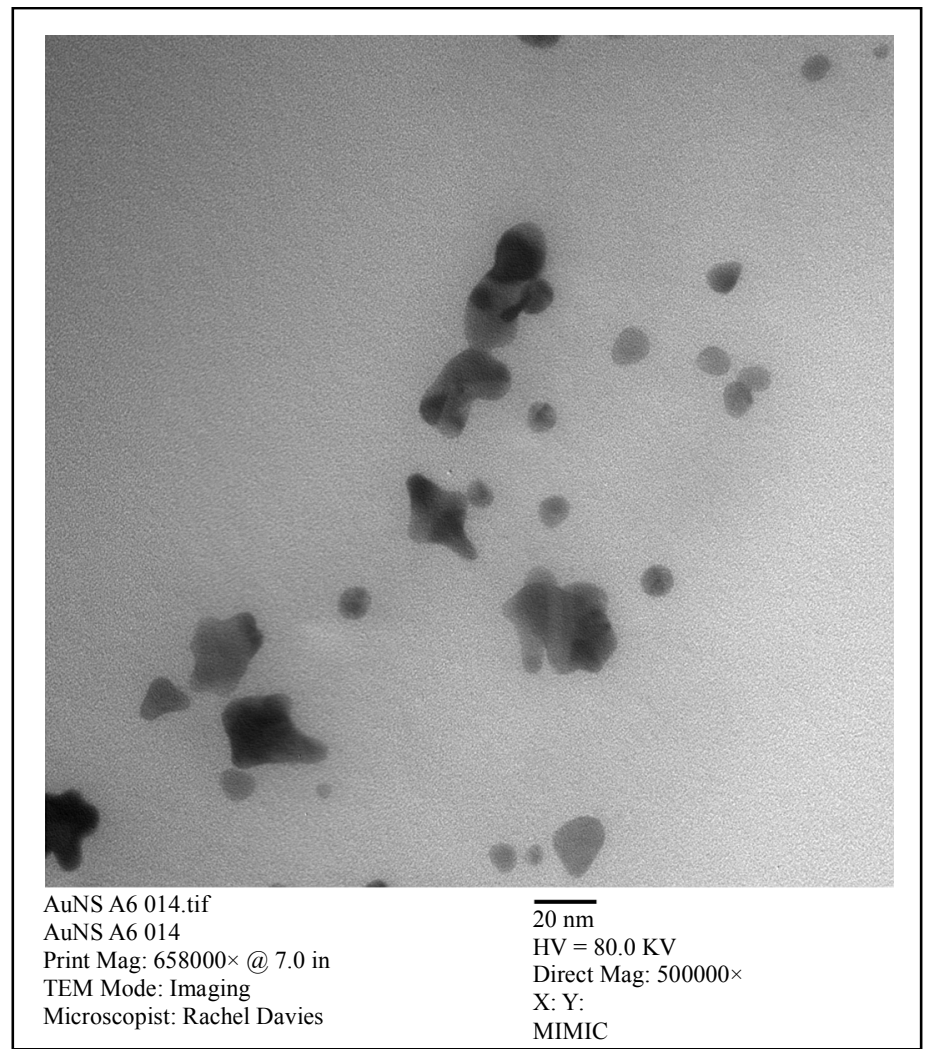

(b)

Figure 6. (a) TEM image of colloidal gold nanostar clusters at 200,000× magnification; (b) TEM image of isolated gold nanostars and nanospheres at 500,000 $\times$ magnification; the black horizontal bar below Image (a) and (b) represents $100 \mathrm{~nm}$ and $20 \mathrm{~nm}$, respectively. 
the nanostars are identified by the arrows. Based on the definition of Khoury and Vo-Dinh [19] in which a nanostar is interpreted as a spherical core of diameter D, from which a number $(\mathrm{N})$ of round-end cone "branches" with the parameters of branch base $\left(\mathrm{D}_{\mathrm{a}}\right)$, branch length (L), and branch tip $\left(\mathrm{D}_{\mathrm{b}}\right)$ originate from the spherical core. The nanostar parameters observed in the colloid prepared in this experimental study are approximately $\mathrm{D}=15-20$ $\mathrm{nm}, \mathrm{L}=10-18 \mathrm{~nm}, \mathrm{D}_{\mathrm{a}}=5-12 \mathrm{~nm}$, and $\mathrm{D}_{\mathrm{b}}=4-6 \mathrm{~nm}$, and $\mathrm{N}=2-4$, which are consistent with previously published values [18]. Among all these parameters, the branch length or $\mathrm{L}$ is the most significant in influencing the SERS intensities since the localized surface plasmon resonance effect is concentrated at the branches, especially their tips. Based on the ratio of nanostars to nanospheres observed in TEM, it is likely that the SERS enhancement can be improved further by varying the concentration of gold (III) chloride solution to maximize the production of nanostars and to increase the length of the nanostar branches.

The nebulization of colloidal samples onto carboncoated grids was found to be a suitable sample preparation method for TEM imaging of metal nanoparticles. The ability to gently deposit the particles without rupturing the carbon film on the TEM grid along with the quick evaporation of the solvent mostly in the gas phase allow the observation of nanoparticles as close to their native state of colloidal dispersion. The variables of the grid exposure time and the distance between TEM grid and nebulizer nozzle have to be controlled for optimal coverage of grid with nanoparticles, which were found to be 40 - 60 seconds and $7-10 \mathrm{~cm}$, respectively.

\subsection{Potential Applications of SERS Technique for Monitoring Anilines}

Anilines are an important class of compounds widely used in industries, especially as a precursor for making dyes for fabric, leather, and other consumer goods. Workers in manufacturing industries involved in the production and use of dyes face occupational risk in being exposed to harmful levels of aromatic amines including anilines. Therefore, a robust and inexpensive method for screening or monitoring these compounds are highly desired for human health protection. Although aniline is the most important member of all the aniline compounds in terms of production volume and toxicological importance, other anilines are also included in this study for a more comprehensive evaluation of the feasibility of the SERS technique for analyzing samples with aniline and aniline derivatives.

The limits of detection (LODs) were calculated for selected ortho-substituted anilines. Since the substituents of these anilines either exert the inductive effect of electron withdrawal from the amino group (i.e. nitro group or halogens) or pose steric hindrance for the charge-transfer bonding via the lone pair of electrons on the nitrogen atom of the amino group, the lower enhancement factors of the ortho-substituted anilines relative to aniline always raise their detection limits relative to aniline. In Table 1, the LOD of aniline using the SERS methodology is estimated at $1.6 \mathrm{ppm}$ for colloidal silver substrate compared to $0.82 \mathrm{ppm}$ for colloidal gold. Other LODs for aniline derivatives are higher than those of aniline because the ortho substituents either pose a steric constraint or inductive effect of electron withdrawal that preclude effective chemisorption of the compounds via the amino group. In general, the LODs are lower for SERS measurement using gold nanostars, which yield LODs in the range of $0.82 \mathrm{ppm}$ to $33 \mathrm{ppm}$, compared to the LOD range of 1.6 ppm to $1700 \mathrm{ppm}$ for silver nanospheres. Compounds such as the di-halogenated anilines as well as the monoand di-nitroanilines exhibit high levels of fluorescence background when silver colloid is used and hence their LOD values are generally higher than the non-fluorescent compounds. Both the enhancement factors (Table 2) and LODs show that the steric hindrance for the bulky tertiarty-butyl group is more significant than the isopropyl group in the ortho position, a notion expressed in the paper by Ikezawa et al. [4]

These LOD values are encouraging in view of the possibility to improve the development of highly sensitive and reproducible SERS substrates as discussed in recent references [10,18-20]. One study reported the use of 3-aminopropyltriethoxysilane for coating nanostar particles onto a glass slide for achieving detection limits of 5 $\times 10^{-11} \mathrm{M}$ and $1 \times 10^{-9} \mathrm{M}$ for Nile blue $\mathrm{A}$ and Rhodamine $6 \mathrm{G}$, respectively. The possibility to couple the technique to an electrochemical apparatus for increasing the adsorption of anilines by biasing a potential of -0.15 volt vs. $\mathrm{Ag} / \mathrm{AgCl}$ reference on a silver-electrodeposited screen-printed electrode is another useful strategy aimed toward the application of SERS for rapid analysis of aromatic amines [8].

\section{Conclusion}

This study is designed to investigate the variables influencing the magnitude of SERS enhancement factors. It was found that gold nanostar is more effective than the silver nanospheres in enhancing the SERS signals of anilines. The greater enhancement efficiency of gold nanostars is due to the smaller energy gap between the HOMO of anilines and the LUMO of the gold surface. The substituents at the ortho position of anilines also lower the $\mathrm{pKa}$ values to different extent such that high enhancement factors are strongly correlated with high 
$\mathrm{pKa}$ values. Electron-withdrawing substituents including the nitro group tend to give minimal SERS enhancement relative to the alkyl substituents. Steric hindrance based on the presence of isopropyl or tertiary butyl groups at the ortho position is secondary to the importance of the $\mathrm{HOMO} / \mathrm{LUMO}$ and $\mathrm{pKa}$ factors. All the isopropyl or tertiary butyl derivatives of anilines yield slightly lower enhancement factors and somewhat higher LODs relative to aniline. The TEM characterization of the gold nanostars corroborates the high degree of aggregation with up to 60 nanoparticles per cluster, thereby providing "hotspots" for large enhancement factors.

\section{Acknowledgements}

The authors wish to express their appreciation to Joyce Miller at the MTSU Interdisciplinary Microanalysis and Imaging Center at Middle Tennessee State University.

\section{REFERENCES}

[1] G. C. Schatz, M. A. Young and R. P. Van Duyne, "Electromagnetic Mechanism of SERS," Topics in Applied Physics, Vol. 103, 2006, pp. 19-46. doi:10.1007/3-540-33567-6_ 2

[2] A. M. Michaels, M. Nirmal and L. E. Brus, "Surface Enhanced Raman Spectroscopy of Individual Rhodamine 6G Molecules on Large Ag Nanocrystals," Journal of the American Chemical Society, Vol. 121, No. 43, 1999, pp. 9932-9939. doi:10.1021/ja992128q

[3] Y. Fan and S. Q. Man, "SERS Study of Malachite Green on Silver Nanocaps Arrays," Advanced Materials Research, Vol. 418-420, 2012, pp. 8-12. doi:10.4028/www.scientific.net/AMR.418-420.8

[4] Y. Ikezawa, H. Saito, K. Matsui and G. Toda, "A Study of the Competitive Adsorption of Pyridine and Monosubstituted Pyridines on a Silver Electrode by the SERS Method," Surface Science, Vol. 176, No. 3, 1986, pp. 603-609. doi:10.1016/0039-6028(86)90058-0

[5] T. Tanaka, A. Nakajim, A. Watanabe, T. Ohno and Y. Ozaki, "Surface-Enhanced Raman Scattering Spectroscopy and Density Functional Theory Calculation Studies on Adsorption of o-, m-, and p-Nitroaniline on Silver and Gold Colloid," Journal of Molecular Structure, Vol. 661662, 2003, pp. 437-449.

[6] M. A. Palafox, J. L. Nunez and M. Gil, "Accurate Scaling of the Vibrational Spectra of Aniline and Several Derivatives," Journal of Molecular Structure (Theochem), Vol. 593, No. 1-3, 2002, pp. 101-131. doi:10.1016/S0166-1280(02)00319-6

[7] H. Park, S. B. Lee, K. Kim and M. S. Kim, "SurfaceEnhanced Raman Scattering of p-Aminobenzoic Acid at Ag Electrode," Journal of Physical Chemistry, Vol. 94, No. 19, 1990, pp. 7576-7580. doi:10.1021/j100382a049

[8] D. Li, D-W. Li, J. D. Fossey and Y. Long, "Portable Surface-Enhanced Raman Scattering Sensor for Rapid Detection of Aniline and Phenol Derivatives by On-Site Electrostatic Preconcentration," Analytical Chemistry,
Vol. 82, No. 22, 2010, pp. 9299-9305. doi:10.1021/ac101812x

[9] N. Leopold and B. Lendl, "A New Method for fast Preparation of Highly Surface-Enhanced Raman Scattering (SERS) Active Silver Colloids at Room Temperature by Reduction of Silver Nitrate with Hydroxylamine Hydrochloride," Journal of Physical Chemistry B, Vol. 107, No. 24, 2003, pp. 5723-5727. doi:10.1021/jp027460u

[10] J. P. Xie, J. Y. Lee and D. I. C. Wang, "Seedless, Surfactantless, High-Yield Synthesis of Branched Gold Nanocrystals in HEPES Buffer Solution," Chemistry of Materials, Vol. 19, No. 11, 2007, pp. 2823-2830. doi:10.1021/cm0700100

[11] L.-B. Zhao, R. Huang, M.-X. Bai, D.-Y. Wu and Z.-Q. Tian, "Effect of Aromatic Amine-Metal Interaction on Surface Vibrational Raman Spectroscopy of Adsorbed Molecules Investigated by Density Functional Theory," The Journal of Physical Chemistry, Vol. 115, No. 10, 2011, pp. 4174-4183.

[12] N. S. Chong, K. A. Smith, S. Setti and B. G. Ooi, “Application of Gold and Silver Colloidal Nanoparticles for the Surface-Enhanced Raman Spectrometric Analysis of Melamine and 4-Aminobiphenyl," International Journal of Environmental Technology and Management, Vol. 16, No. 1/2, 2013, pp. 3-20. doi:10.1504/IJETM.2013.050681

[13] L.-B. Zhao, Y.-F. Huang, X.-M. Liu, J. R. Anema, D.-Y. Wu, B. Ren and Z.-Q. Tian, "A DFT Study on Photoinduced Surface Catalytic Coupling Reactions on Nanostructured Silver: Selective Formation of Azobenzene Derivatives from para-Substituted Nitrobenzene and Aniline," Physical Chemistry Chemical Physics, Vol. 14, 2012, pp. 12919-12929. doi:10.1039/c2cp41502j

[14] K. A. Smith, N. S. Chong, K. Donthula and B. G. Ooi, "Influence of the Structural Characteristic of Silver and Gold Nanoparticles on the Surface-Enhancement Factors of the Raman Signals from Aromatic Amines," NanoFormulation, Series 336, 2012, pp. 121-132.

[15] J. T. Hugall, J. J. Baumberg and S. Mahajan, "Disentangling the Peak and Background Signals in Surface-Enhanced Raman Scattering," Journal of Physical Chemistry C, Vol. 116, No. 10, 2012, pp. 6184-6190. doi:10.1021/jp3002977

[16] L. Lipping, A. Kutt, K. Kaupmees, I. Koppel, P. Burk, I. Leito and I. A. Koppel, "Acidity of Anilines: Calculations vs. Experiment," Journal of Physical Chemistry A, Vol. 115, No. 37, 2011, pp. 10335-10344. doi:10.1021/jp204064q

[17] K. C. Gross and P. G. Seybold, "Substituent Effects on the Physical Properties and pKa of Aniline," International Journal of Quantum Chemistry, Vol. 80, No. 4-5, 2000, pp. $1107-1115$. doi:10.1002/1097-461X(2000)80:4/5<1107::AID-QUA60 3.0.CO;2-T

[18] E. N. Esenturk and A. R. H. Walker, "Surface-Enhanced Raman Scattering Spectroscopy via Gold Nanostars" Journal of Raman Spectroscopy, Vol. 40, No. 1, 2009, pp. 86-91. doi:10.1002/jrs.2084

[19] C. G. Khoury and T. Vo-Dinh, "Gold Nanostars for Surface-Enhanced Raman Scattering: Synthesis, Characteri- 
zation and Optimization" Journal of Physical Chemistry C, Vol. 112, No. 48, 2008, pp. 18849-18859.

[20] Q. Su, X. Ma, J. Dong, C. Jiang and W. Qian "A Reproducible SERS Substrate Based on Electrostatically As- sisted APTES-Functionalized Surface-Assembly of Gold Nanostars," ACS Applied Materials \& Interfaces, Vol. 3, No. 6, 2011, pp 1873-1879. doi:10.1021/am200057f 